Low-temperature Swelling in LWR Internal Components:

Current Data and Modeling Assessment

R. E. Stoller, A. V. Barashev, and S. I. Golubov

Materials Science and Technology Division

Oak Ridge National Laboratory

Prepared for:

Light Water Reactor Sustainability Program

Office of Nuclear Energy, Science and Technology

U.S. Department of Energy

September 2012 


\section{Low-temperature Swelling in LWR Internal Components: Current Data and Modeling Assessment}

\section{Abstract}

Recent experimental observations have made it clear that cavity formation can occur in light-water reactor internal components fabricated from austenitic stainless during the course of their service life. In order to assess the potential for cavity swelling in these components at end-of-life doses, it is necessary to develop a validated computational model that incorporates the relevant physical mechanisms and accounts for recent experiment data. Such a modeling activity is underway; the model development and some preliminary results are described. For the relatively low temperatures involved, cavity formation is shown to be sensitive to both the temperature and the rate of helium production by nuclear transmutation reactions. This report includes a brief review of the relevant microstructural data, discussion of the current model's status and planned further development, and a description of the microstructural modeling that is planned to fully define the potential for cavity evolution under light water reactor operating conditions.

\section{Introduction}

The phenomenon of void swelling was first reported by Cawthorne and Fulton in the late 1960s [1,2] when the development of fast breeder reactors (FBR) was the focus of many national energy programs. For almost twenty years, a dominant focus of radiation effects research was the pursuit of understanding what caused void swelling and the development of possible strategies to prevent or mitigate it [3]. Since austenitic stainless steels were the prime candidate for use as fuel cladding and duct in these sodium cooled reactors, a substantial experimental database was developed on these materials. However, this database was subject to the limitations imposed by both the target FBR technology and the sodium-cooled test reactors that were used to obtain the data. The sodium temperature in the FBR was typically about $350^{\circ} \mathrm{C}$ at the inlet to the core, and this naturally sets the minimum temperature of the swelling data that was obtained. In addition, the axial variation in neutron flux meant that the lower temperature data was obtained at a lower flux and was therefore limited to lower total fluences than data from further into the core where the exposure temperature was higher. Mechanical performance limits of these materials limited the upper temperature of the swelling experiments to $\sim 650^{\circ} \mathrm{C}$. Thus, the preponderance of the high fluence swelling data obtained in the FBR programs lay in the temperature range of 400 to $600^{\circ} \mathrm{C}$. The overall picture of the temperature and neutron fluence dependence of swelling that developed is illustrated in Fig. 1 [4,5]. Although there is a microstructural and mechanistic basis for the view that swelling is reduced at the lowest and highest temperatures, the inference that swelling should not exist below about $350^{\circ} \mathrm{C}$ was not founded on a broad body of high fluence data. In particular, the implications of the significant increase in the incubation time for visible swelling at low temperatures may have been overlooked.

Because of the temperature limitations just mentioned, the FBR database cannot be directly applied to predict the swelling performance of austenitic steels in light water 

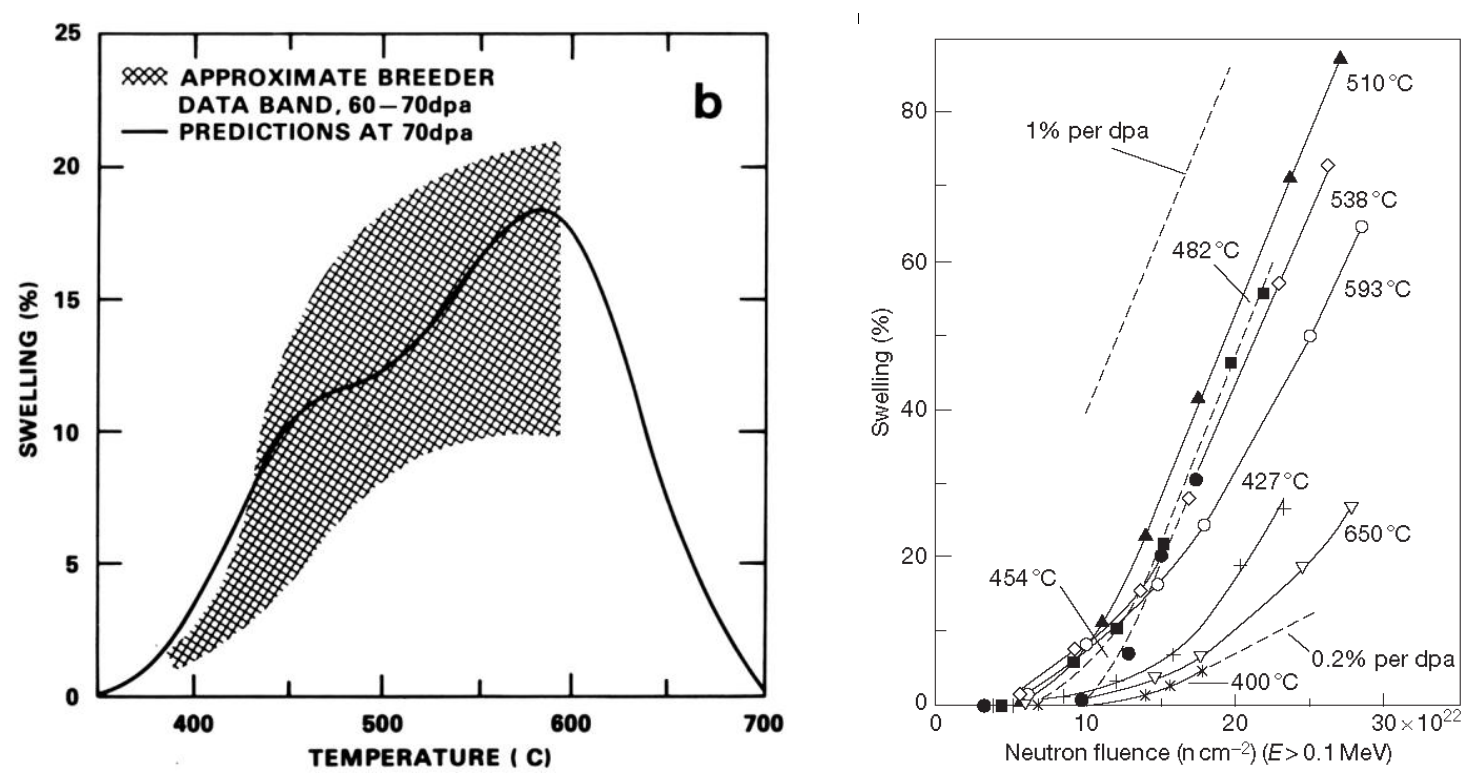

Fig. 1 Illustration of the temperature and neutron fluence dependence of representative fast reactor swelling data $[4,5]$.

reactor (LWR) internal components because these components operate in a nominal temperature range of 270 to $300^{\circ} \mathrm{C}$. However, the FBR results have strongly influenced the expectations of LWR operators and they have not anticipated any swelling to occur during service. As will be discussed in the next section, the assumption of no swelling LWR internals appears unwarranted. Although swelling was minimal at $\sim 350^{\circ} \mathrm{C}$, the maximum neutron fluence was also typically low at these conditions where swelling incubation time was longest. With the advent of license extensions for commercial LWRs, the maximum expected neutron exposure is now sufficiently high to raise concerns about exceeding the swelling incubation time [5].

Before proceeding to discuss relevant data and the current models, it is useful to define a few terms that will be used in this report. The evolution of the cavity microstructure in irradiated materials begins with the formation of small vacancy clusters. The cavities grow by absorbing vacancies and shrink by vacancy emission and interstitial absorption. For very small cavities the dominant process is thermodynamically determined to be vacancy emission. Statistical fluctuations can still produce large vacancy clusters since having vacancy clusters of size $(n)$ and mobile vacancies implies a non-zero probability of having (fewer) clusters of size $(n+1)$ which can in turn yield clusters of size $(n+2)$ and so on. However, homogeneous nucleation rates are computed to be several orders of magnitude too small to explain the observed cavity densities [6]. Cavity nucleation is aided by residual gases (e.g., $\mathrm{N}_{2}$ and $\mathrm{O}_{2}$ ) in the material from the melt and by transmutant gases produced under irradiation. Both helium and hydrogen are produced in stainless steel by $(n, \alpha)$ and $(n, p)$ reactions, respectively. Because helium is insoluble in the atomic lattice, it is strongly trapped by vacancy defects [7].

In this context the terms cavity, bubble, and void can be defined for the purposes of this report. Cavity is used in a general way to describe any accumulation of 
apparently empty volume in the metal matrix; this volume may comprise both vacancies and gas atoms. A cavity which is primarily stabilized by the gas atoms it contains is referred to as a bubble. The gas pressure in a bubble of radius $r$ at temperature $T$ is a significant fraction of the equilibrium value of $2 \gamma / r$, where $\gamma$ is the free surface energy. A cavity which is primarily an agglomeration of vacancies has $\mathrm{P}<<2 \gamma / \mathrm{r}$ and is called a void. The bubbles produced in reactor irradiations tend to be small, $r<2.5 \mathrm{~nm}$, while void radii may range up to several hundred nanometers or more. Bubbles tend to be spherical, while the larger voids are frequently faceted. Voids are not stable under post-irradiation annealing conditions and will disappear or shrink back to a bubble of the size appropriate to their gas content.

As shown in Fig. 1b, the time dependence of the cavity evolution or swelling tends to exhibit three fairly distinct regions. There is an initial period associated with the formation of a sub-critical cavity (bubble) population. During this period there is little density change and the cavities may remain nearly invisible under transmission electron microscopy (TEM) examination (i.e., $r<0.5 \mathrm{~nm}$ ). Next there is a transient which is a result of some of the subcritical bubbles reaching a critical radius and beginning to grow as voids $[6,8]$. At this point the cavities are visible under TEM and the reduction in density due to the void volume is measurable but generally less than $1 \%$. Finally a regime of "steady state" swelling is reached as those voids which have previously nucleated grow rapidly by vacancy absorption. This regime is characterized by high swelling rates, $\sim 1 \% / \mathrm{dpa}$, and a bi-modal cavity size distribution with small subcritical bubbles and large voids can frequently be observed [3].

Although the familiar terms neutron flux and fluence have been used above, the neutron exposure parameter used in the succeeding discussion will be the number of atomic displacements that have been generated per lattice atom (dpa or dpa rate). The use of dpa, which is a measure of kinetic energy absorbed by the material, provides a better physical basis for comparing the damage potential of either reactors with different neutron energy spectra or neutron with charged particle irradiation [9]. When discussing the influence of transmutation produced gas, it is convenient to deal with the ratio of gas production to displacement production in units of appm gas per dpa.

\section{Background and Data Assessment}

In addition to the difference in exposure temperature between the irradiation environment of FBR and LWR core components already mentioned, the neutron energy spectrum is also quite different. This is shown in Fig. 2 which displays the neutron energy spectrum in the core of Fast Flux Test Facility (FFTF), the central flux trap of the High Flux Isotope Reactor (HFIR), a typical pressurized water reactor (PWR) within the pressure vessel, and a deuterium-tritium fusion reactor first wall [10]. Although the HFIR flux is much higher than the PWR, the energy spectrum is quite similar because both reactors are water moderated and cooled. The FFTF was a sodium-cooled reactor which leads to a "harder" neutron spectrum, i.e. a much greater fraction of the neutrons remain at high energies with relatively few neutrons below $1 \mathrm{eV}$. This difference is significant because the thermal neutrons can lead to the production of substantial amounts of helium in a nickel-bearing austenitic steel. The helium is produced by 


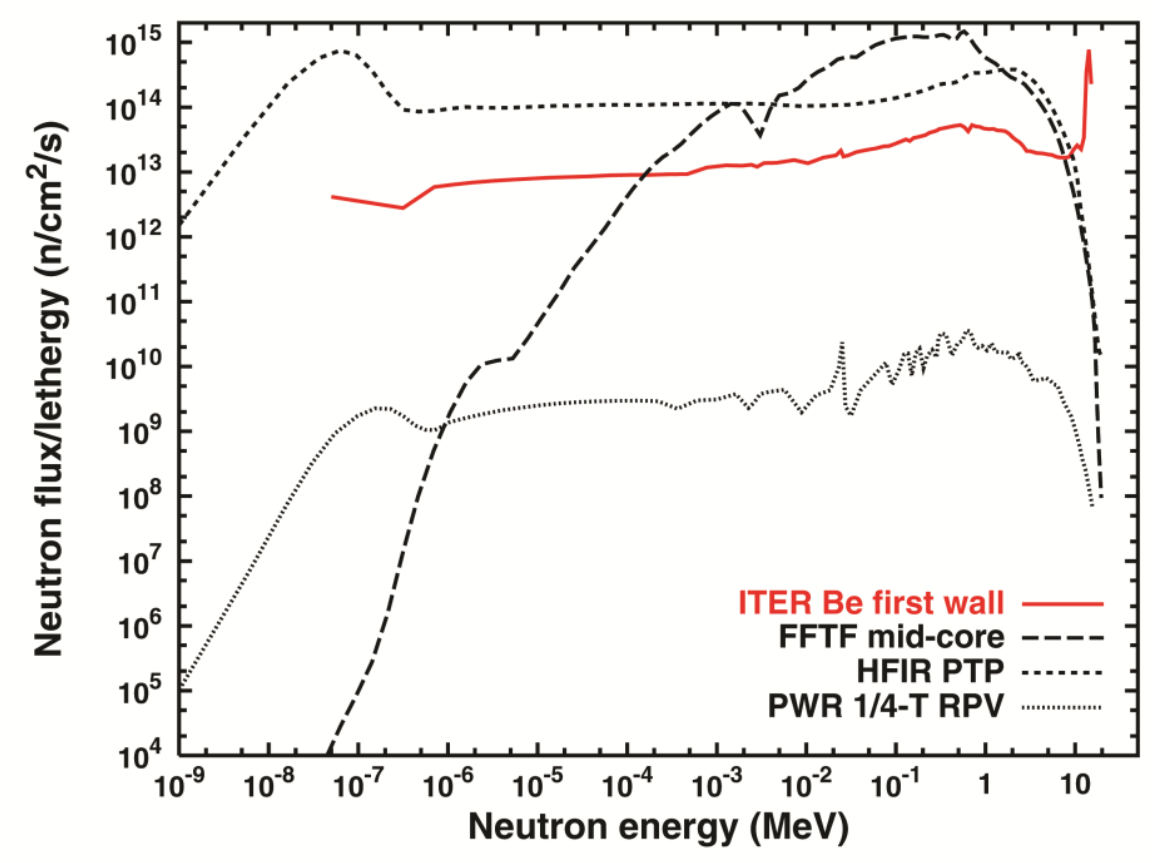

Fig. 2. Comparison of neutron energy spectra from different reactors[10].

thermal neutrons in a two-step reaction:

$$
{ }^{58} \mathrm{Ni}(\mathrm{n}, \gamma){ }^{59} \mathrm{Ni}(\mathrm{n}, \alpha){ }^{56} \mathrm{Fe} .
$$

This He production is nonlinear in time due to the buildup of ${ }^{59} \mathrm{Ni}$. For example, the $\mathrm{He}$ production rate in the core of the HFIR is initially $<1$ appm He/dpa and eventually saturates at $\sim 80 \mathrm{appm} \mathrm{He/dpa} \mathrm{in} \mathrm{a} \mathrm{typical} \mathrm{stainless} \mathrm{steel.} \mathrm{The} \mathrm{lack} \mathrm{of} \mathrm{thermal} \mathrm{neutrons}$ in an FBR leads to a lower and constant He/dpa ratio which is typically 0.1 to 0.3 appm/dpa. Because helium can enhance cavity nucleation, this difference also suggests that the FBR swelling database may be non-conservative for predicting swelling in a LWR core.

A related difference between the LWR and FBR environments is the level of volumetric heating due to gamma ray absorption. Although the fission-generated gamma fluxes would be similar in a fast and thermal reactor operating at the same fission rate, the higher thermal flux in a water cooled reactor would lead to substantial gamma ray generation by thermal neutron capture reactions. For example, the reported gamma heating rates in stainless steel are $46 \mathrm{w} / \mathrm{g}$ in the HFIR [11] and $5.6 \mathrm{w} / \mathrm{g}$ in the sodium cooled Experimental Breeder Reactor-II [12]. The increase in volumetric heating due to the higher gamma flux could significantly increase the internal temperature of thick components to well above their nominal surface temperature. This would increase the likelihood of void swelling.

A final difference between the FBR and LWR environments is the damage rate; in typical fast reactor irradiations the rate is about $1 \times 10^{-6} \mathrm{dpa} / \mathrm{s}$ while for LWR core components is about $5 \times 10^{-8} \mathrm{dpa} / \mathrm{s}$. Unfortunately, the effects of displacement rate are 
difficult to separate from temperature effects both experimentally and computationally. In an FBR core, the displacement rate increases from the bottom to the mid-plane and then decreases. The coolant temperature continually increases. In the case of fuel pins, which provided much of the FBR swelling data, it is difficult to obtain iso-temperature, iso-displacement-rate swelling measurements because of the co-variance of these parameters. However, it has been experimentally observed that lower displacement rates tend to reduce the length of the low-swelling incubation regime [3a], and this same effect has been observed in computational modeling [13]. The data is somewhat ambiguous regarding the temperature range over which this reduction occurs [13], but if the behavior persists to $\sim 300^{\circ} \mathrm{C}$ this could be another example of how predictions based on FBR data could be non-conservative for LWR components. Any effect of displacement rate will confound direct comparisons between some PWR and boiling water reactor (BWR) components since the latter are generally exposed at lower rates.

Since about the mid-1990s there has been increasing discussion of the likelihood of swelling in LWR components [14]. Post-irradiation examination of core components has provided many examples of cavity formation, although the level of swelling remains small for the exposure conditions examined so far. Garner provided a thorough review of the relevant data in a 2010 article [5]. Examples include small cavities in a BWR shroud assembly after irradiation to only $1.9 \mathrm{dpa}$ at $290^{\circ} \mathrm{C}$ [15], and larger voids in a baffle bolt taken from the Tihange pressurized water reactor (PWR) in Belgium [16]. In this latter example, irradiation to $12 \mathrm{dpa}$ at an estimated temperature of $345^{\circ} \mathrm{C}$ had led to $0.2 \%$ swelling. The elevated temperature relative to the reactor coolant is a result of the gamma heating discussed above. An example of the voids observed during TEM examination of the Tihange baffle bolt is shown in Fig. 3. Edwards and co-workers also examined two sections cut from a PWR thimble tube after 23 years of service; one section was exposed at $290^{\circ} \mathrm{C}$ to $33 \mathrm{dpa}$ and the other at $315^{\circ} \mathrm{C}$ to $70 \mathrm{dpa}$ [17]. Small bubbles $(\mathrm{r}<3 \mathrm{~nm})$ were observed at a density of $\sim 10^{23} \mathrm{~m}^{-3}$.

A substantial body of relevant data has been obtained from post-irradiation

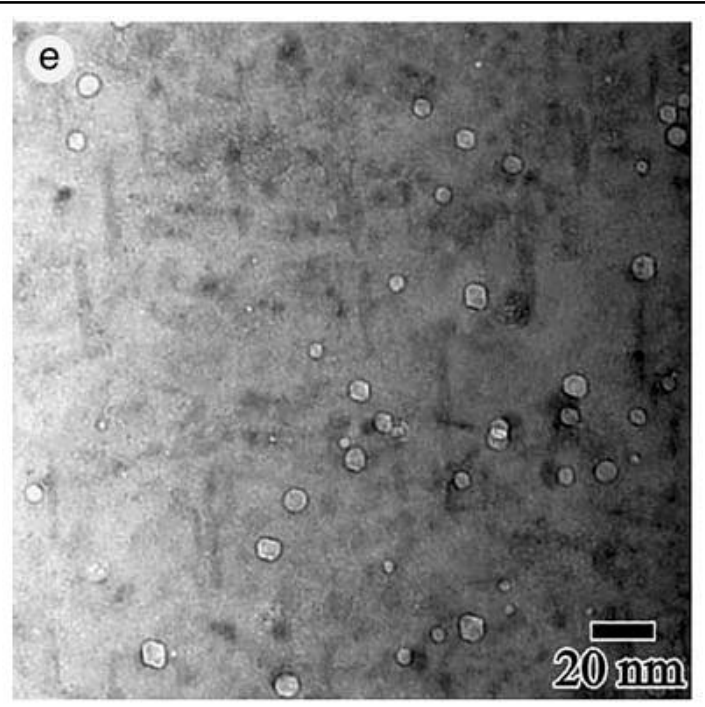

Fig. 3. Voids observed during TEM examination of baffle bolt from Tihange reactor in Belqium [171. 
examination of components from Russian reactors [5]. From this data, a final example is shown in Fig. 4 which was obtained by TEM examination of a Russian austenitic stainless steel, $12 \mathrm{X} 18 \mathrm{H} 9 \mathrm{~T}\left((0.12 \mathrm{C}-18 \mathrm{Cr}-9 \mathrm{Ni}-\mathrm{Ti})\right.$ irradiated at $\sim 350^{\circ} \mathrm{C}$ in the BR-10 fast reactor to a very low dose of $0.64 \mathrm{dpa}$ [18]. The dose rate was also quite low, $\sim 1.9 \times 10^{9}$ $\mathrm{dpa} / \mathrm{s}$. The mean void diameter was $11 \mathrm{~nm}$, although voids with diameters up to almost $20 \mathrm{~nm}$ can be seen in the figure. The concentration was $\sim 6 \times 10^{20} \mathrm{~m}^{-3}$, leading to about $0.1 \%$ swelling.

There is a sufficient number of experimental observations of cavities with large enough sizes and high enough densities to confirm that measurable (if small) swelling is already occurring under LWR conditions. Such data make a compelling case for the need to develop a predictive model of swelling under these conditions in order to anticipate how much swelling may occur as the operating life of the current fleet of reactors is extended. It is essentially impossible to follow the approach taken by the fast
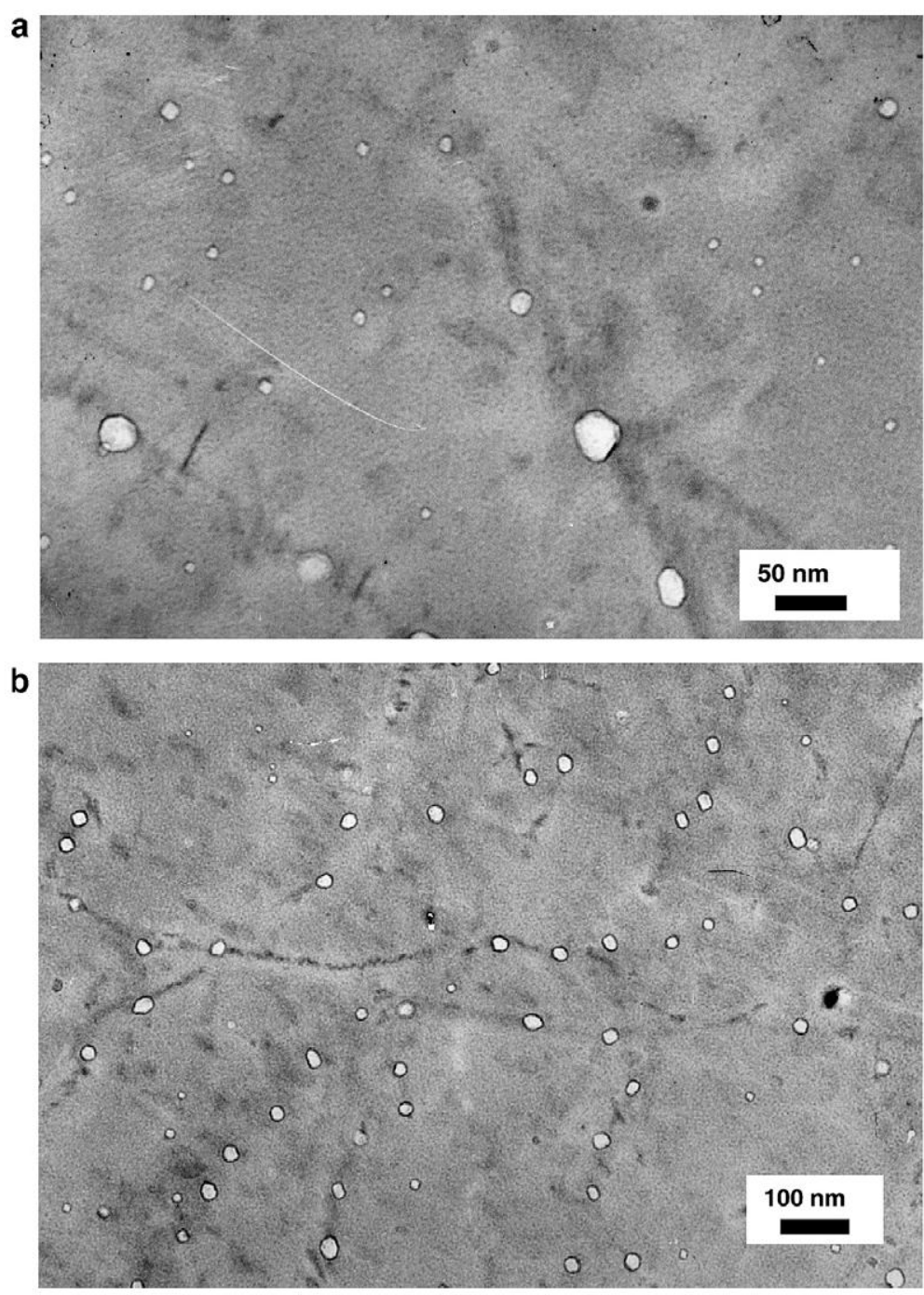

Fig. 4. Voids observed during TEM examination of a Russian $18 \mathrm{Cr}-9 \mathrm{Ni}-\mathrm{Ti}$ steel irradiated to $0.64 \mathrm{dpa}$ at $\sim 350^{\circ} \mathrm{C}[18]$. 
reactor program in the 1970s and 1980s, which involved massive irradiation experiments to obtain data to fit an empirical swelling correlation. The development and validation of a mechanistically sound microstructural model is proposed.

\section{Model Development and Initial Results}

The basis for the new model is a comprehensive microstructural model that was developed by the principle investigator for similar materials in fast reactor fuel cladding. $[4,19]$. The model employs the well-known reaction rate theory description of radiation damage formation and damage evolution, and explicitly accounts for the evolution of both the dislocation and cavity microstructures. The dislocation component includes the formation and growth of small interstitial loops which are known to be important at low to intermediate temperatures. The impact of helium produced by nuclear transmutation is observed in the nucleation voids from an initial distribution of sub-critical bubbles.

Several areas have been identified in which the base model requires revision and further development to accurately describe the LWR environment; the principle areas are:

1. modify the description of primary radiation damage formation to account for recent advances in understanding based on atomistic simulations of damage production, i.e. point defect survival and clustering and interstitial cluster mobility [20],

2. provide an explicit bubble nucleation component based on a cluster dynamics description of He-vacancy clustering.

3. revise the reaction-diffusion components of the model to account for additional diffusion mechanisms and the relevant sink strengths for extended defects, i.e. mixed one- and three-dimensional diffusion of small interstitial clusters [21],

4. modification of the primary radiation damage source terms in terms of displacements per atom (dpa) and helium production to account for the neutron energy spectrum, and

5. implement a recently developed approach for discretizing the interstitial loop size distribution to obtain greater accuracy.

Of the items in this list, numbers (1) to (3) are particularly significant. The initial model did not account for cavity nucleation explicitly. Based on microstructural data in the literature, a population of sub-critical bubbles was assumed to be present at the start of the simulations. This fixed density of bubbles could grow by helium and vacancy accumulation until they reached the critical size for bubble-to-void conversion $[6,8]$. Above this size, they began to grow more rapidly as voids. The weakness of this approach was the empirical (if experimentally guided) selection of the initial bubble population. The dose required for bubble nucleation is not accounted for in this scheme and it may become increasingly long for the low LWR temperatures. In addition, the model could not account for a realistic bubble size distribution. Therefore, a new cluster dynamics description of He-vacancy clustering has been developed to assess bubble nucleation. It can currently be used in a stand-alone fashion to compute the bubble size 
distribution at low doses, and will be incorporated into the comprehensive microstructural model. A few details on this model are given below.

Items (1) and (3) on the list are expected to be important for accurately describing the lower-temperature LWR irradiation conditions in which the density of small cascadeinduced vacancy clusters, and hence their sink strength, is significantly higher than observed at the higher irradiation temperatures typical of FBR components. When the original model is used at temperatures below $\sim 350^{\circ} \mathrm{C}$, these transient vacancy clusters lead to an unphysical suppression of small interstitial dislocation. The high sink strength may also reduce bubble growth. Therefore, improving aspects of both primary radiation damage source terms and the kinetics of small interstitial clusters should lead to a more accurate description of the irradiated microstructure and its evolution at low temperatures.

\section{Bubble nucleation model}

The bubble nucleation model employs an extension of the accurate groping scheme developed by Golubov and co-workers [22] which has been successfully used to simulate bubble evolution under post-irradiation annealing [23]. Briefly, the method solves the two-dimensional master equation describing He-vacancy clusters in a phase space defined by the variables $x$ and $m$, which are the number of vacancies and helium atoms, respectively, in a cluster. We follow the commonly accepted assumption that it is sufficient to assume that the cluster of size $x, m$ can change its size only by absorption and emission of monomers, e.g. single vacancies, self-interstitial atoms, or He atoms. In this case the master equation can be written as:

$$
\frac{d f(x, m, t)}{d t}=\left[J_{x}(x-1, m, t)-J_{x}(x, m, t)\right]+\left[J_{m}(x, m-1, t)-J_{m}(x, m, t)\right],
$$

where

$$
\begin{aligned}
& J_{x}(x, m, t)=P_{x}(x, t) f(x, m, t)-Q_{x}(x+1, m, t) f(x+1, m, t), \\
& J_{m}(x, m, t)=P_{m}(x, t) f(x, m, t)-Q_{m}(x, m+1, t) f(x, m+1, t),
\end{aligned}
$$

and the coefficients $P_{x}(x, t), Q_{x}(x, m, t), P_{m}(x, t), Q_{m}(x, m, t)$ are the rates of capture and evaporation reactions between the monomers and clusters leading to a change in the size of clusters given by $x$ and $m$.

In principle, it is possible to solve Eqn. (2) as an arbitrarily large set of discrete differential equations in the variables $x$ and $m$. However, for bubble sizes typically observed experimentally, this would require a system of millions of equations because the number of equations must equal the product $(x \cdot m)$. For example, a bubble radius of $2.0 \mathrm{~nm}$ requires the maximum value of $\mathrm{x}=3073$ with a similar value of $\mathrm{m}$. Practicable solutions of Eqn. (2) for relevant cases requires developing a scheme for grouping the discrete size classes beyond a value of $x=m \sim 10$. The grouping method used here has been shown to be highly accurate, conserving both the density of the extended defects (bubbles) in the size distribution, and the number of point defects contained in those the bubbles or voids (swelling). The method has been verified by comparison with exact analytical solutions for several cases $[22,23]$. An example of the methods capability is 


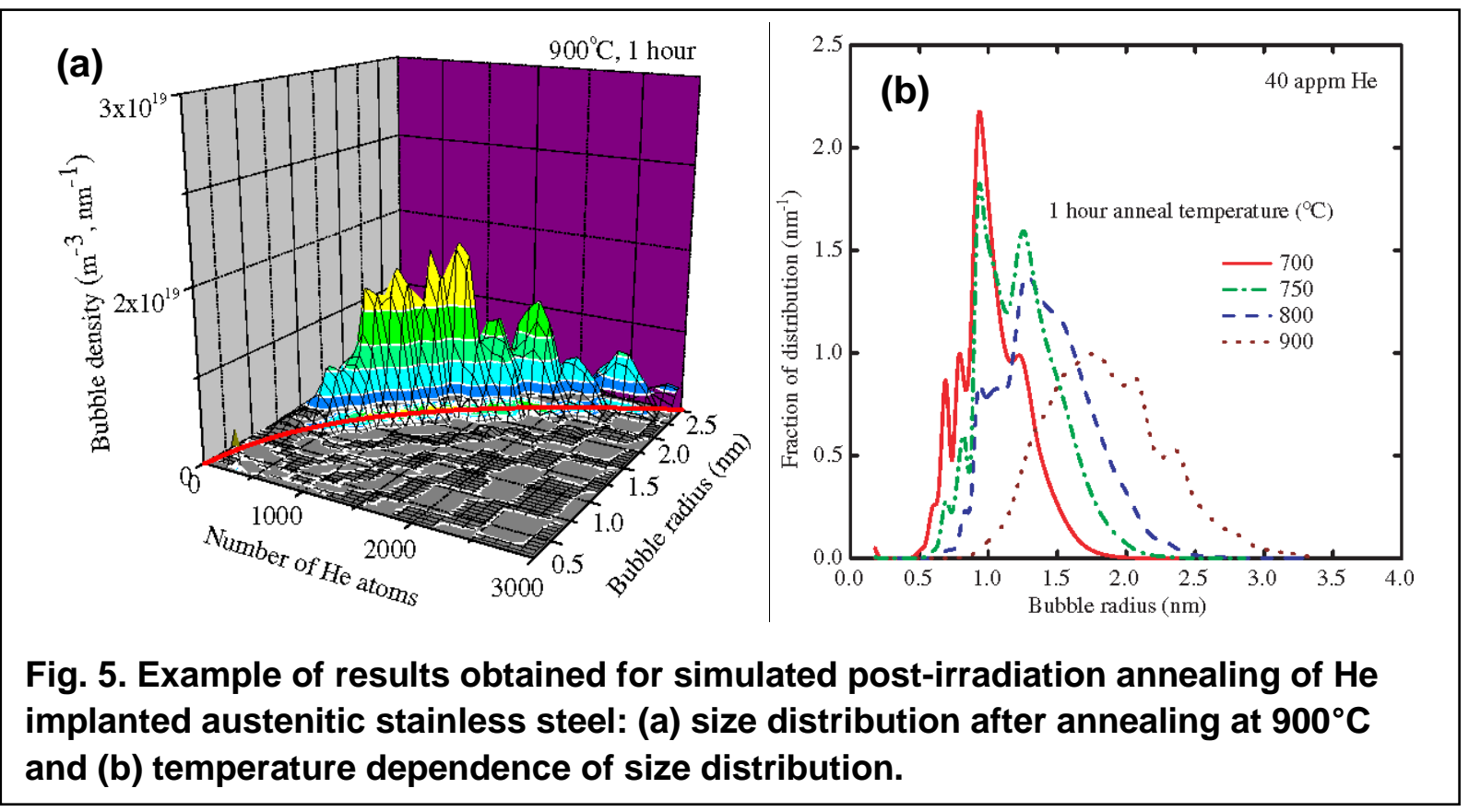

shown in the results taken from Ref. [23] is Fig. 5.

Results of initial calculations

Initial benchmarking runs have been carried out with the original comprehensive microstructural model, and cluster-dynamics-based bubble nucleation model. Material parameters were representative of austenitic stainless steels as used previously $[4,6,19]$ and shown in Table 1. A PWR-relevant atomic displacement rate of $5 \times 10^{-8} \mathrm{dpa} / \mathrm{s}$ was employed and the helium generation rate was varied over a small range that is representative of values obtained at modest doses while the ${ }^{59} \mathrm{Ni}$ content is increasing as discussed above (see discussion of Eqn. (1)). The irradiation temperature range from 275 to $325^{\circ} \mathrm{C}$ was examined. Simulations using the comprehensive model covered up to $30 \mathrm{dpa}$, which is about 20 effective full power years at the specified damage rate. Because there is no microstructural evolution included in the bubble nucleation code other than the bubbles themselves at this time, calculations using this model were limited to $5 \mathrm{dpa}$ to illustrate the sensitivity of nucleation to $\mathrm{He} / \mathrm{dpa}$ ratio an $300^{\circ} \mathrm{C}$.

The results obtained with the bubble nucleation model for irradiation at $300^{\circ} \mathrm{C}$ are shown in Fig. 6, where the fluence dependence of bubble swelling is shown in part (a), the bubble density in part (b), and the mean bubble size in part (c). Values are shown for three different helium generation ratios, 0.5, 1.0 and $2.0 \mathrm{appm} \mathrm{He/dpa.} \mathrm{A} \mathrm{strong}$ sensitivity to the He/dpa ratio is clearly seen, and the tendency toward increasing swelling with dose is visible at only five dpa even for the lowest $\mathrm{He} / \mathrm{dpa}$ ratio. Although the absolute values of the predicted swelling are low, extrapolation to end-of-life doses would lead to substantial swelling. 
ORNL/LTR-2012/390

\begin{tabular}{|c|c|c|c|}
\hline $\begin{array}{l}\text { atomic } \\
\text { displacement rate }\end{array}$ & $5 \times 10^{-8} \mathrm{dpa} / \mathrm{s}$ & surface free energy & $2.25 \mathrm{~J} / \mathrm{m}^{2}$ \\
\hline $\begin{array}{l}\text { cascade survival } \\
\text { efficiency }\end{array}$ & 0.333 & initial dislocation density & $3 \times 10^{15} \mathrm{~m}^{-2}$ \\
\hline $\begin{array}{l}\text { in-cascade vacancy } \\
\text { clustering fraction }\end{array}$ & 0.6 & $\begin{array}{l}\text { dislocation-interstitial } \\
\text { bias }\end{array}$ & 1.25 \\
\hline $\begin{array}{l}\text { vacancy formation } \\
\text { energy }\end{array}$ & $1.6 \mathrm{eV}$ & $\begin{array}{l}\text { dislocation-interstitial } \\
\text { bias }\end{array}$ & 1.25 \\
\hline $\begin{array}{l}\text { vacancy migration } \\
\text { energy }\end{array}$ & $1.4 \mathrm{eV}$ & \multicolumn{2}{|c|}{ Parameters varied: } \\
\hline $\begin{array}{l}\text { interstitial migration } \\
\text { energy }\end{array}$ & $0.85 \mathrm{eV}$ & irradiation temperature & $275,300,325^{\circ} \mathrm{C}$ \\
\hline $\begin{array}{l}\text { recombination } \\
\text { coefficient }\end{array}$ & $\begin{array}{l}2 \times 10^{17} * \text { interstitial } \\
\text { diffusivity }\end{array}$ & helium generation rate & $\begin{array}{l}0.1,0.5,1.02 .0 \\
\text { appm He/dpa }\end{array}$ \\
\hline
\end{tabular}

The temperature and $\mathrm{He} / \mathrm{dpa}$ ratio dependence of the swelling predictions obtained with the comprehensive microstructural model are shown in two cross-plots in Fig. 7. A strong dependence on both variables is clearly observed. The greatest relative change in swelling in Fig 7(a) occurs for a modest incremental change in the He/dpa ratio from 0.1 to 0.5 . This sensitivity to helium is significant because only in a very hard neutron energy spectrum would the value be as low as $0.1 \mathrm{appm} \mathrm{He/dpa} \mathrm{for} \mathrm{an} \mathrm{alloy}$ with several percent nickel. The soft LWR spectrum and the burn in of ${ }^{59} \mathrm{Ni}$ suggest that He production will drive cavity formation in core internals. The effect of temperature is relatively weak only for the very low 0.1 appm He/dpa generation rate.

Figure 8 compares the fluence dependence of swelling obtained with the more comprehensive microstructural model for doses up to $30 \mathrm{dpa}$. Results are shown for irradiation temperatures of $275^{\circ} \mathrm{C}$ in (a), $300^{\circ} \mathrm{C}$ in (b) and $325^{\circ} \mathrm{C}$ in (c). In each graph, values are shown for $\mathrm{He} / \mathrm{dpa}$ ratios of $0.1,0.5,1.0$ and $2.0 \mathrm{appm} \mathrm{He/dpa.} \mathrm{The} \mathrm{swelling}$ shown at $5 \mathrm{dpa}$ for a $300^{\circ} \mathrm{C}$ irradiation temperature in Fig. $8 \mathrm{~b}$ is lower than that obtained with the bubble nucleation model shown in Fig. 6(a). While neither set of values can be considered "correct" since both models have their own limitations, this difference is likely due to the limitations of the cavity formation approach used in the comprehensive model as discussed above. This difference emphasizes the need to incorporate the more detailed cluster dynamics model into the comprehensive model code. The abrupt changes seen in some of the curves (e.g. for $2 \mathrm{appm} \mathrm{He} / \mathrm{dpa}$ at $275^{\circ} \mathrm{C}$ ) are a result of the bubble-to-void conversion process discussed above. In each case, some fraction of the bubbles have converted and their growth rate increases. 

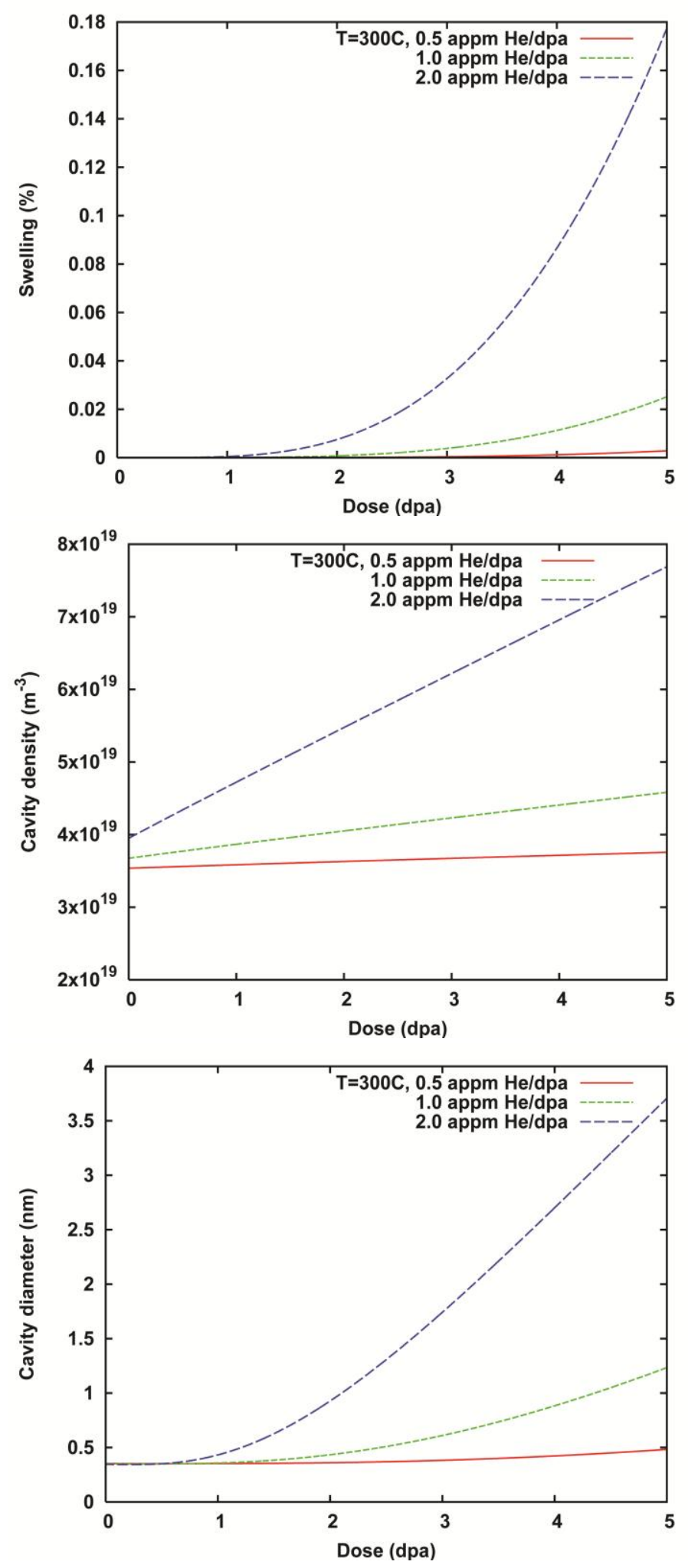

Fig. 6. Predicted dependence of bubble swelling (a), bubble density (b), and mean bubble diameter (c) on the He/dpa ration for low-dose irradiation at $300^{\circ} \mathrm{C}$. 

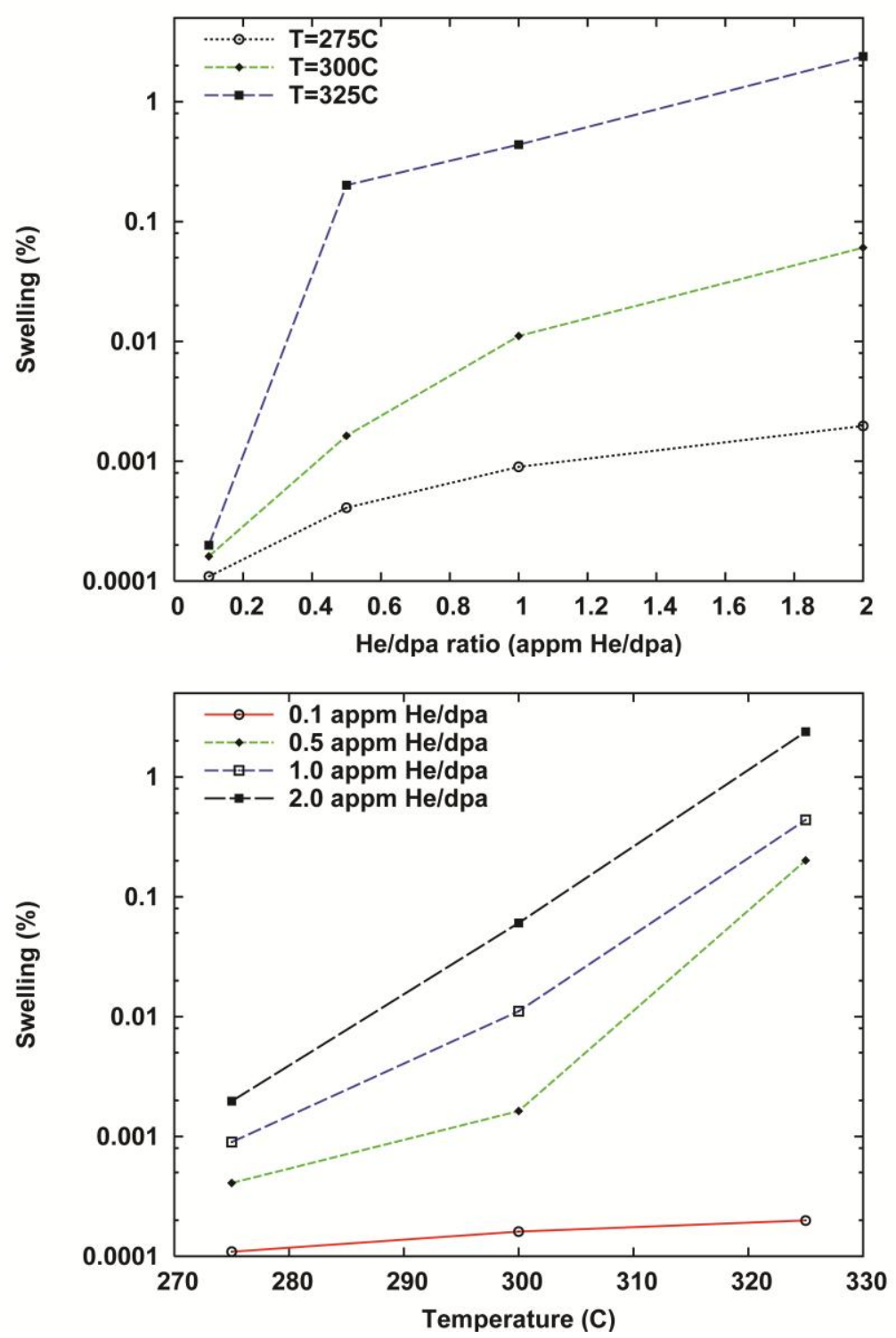

Fig. 7. Parametric cross-plot of predicted swelling at 30 dpa showing sensitivity to temperature and $\mathrm{He} / \mathrm{dpa}$ ratio.

\section{Summary}

Both the available data discussed above and the initial calculations carried out as part of this project indicate that swelling levels of at least a few percent are likely to occur in LWR core components fabricated using austenitic stainless steels. The swelling level is expected to increase for higher temperatures and higher nickel contents since the latter leads to higher helium generation rates. In addition, because of the build-up of ${ }^{59} \mathrm{Ni}$ due to transmutation, the helium generation rate will increase with dose. This will have a non-linear effect on the swelling incubation time and could make swelling predictions based on low-dose observations non-conservative. The dependence of the swelling incubation time on displacement rate has not been definitively established in 

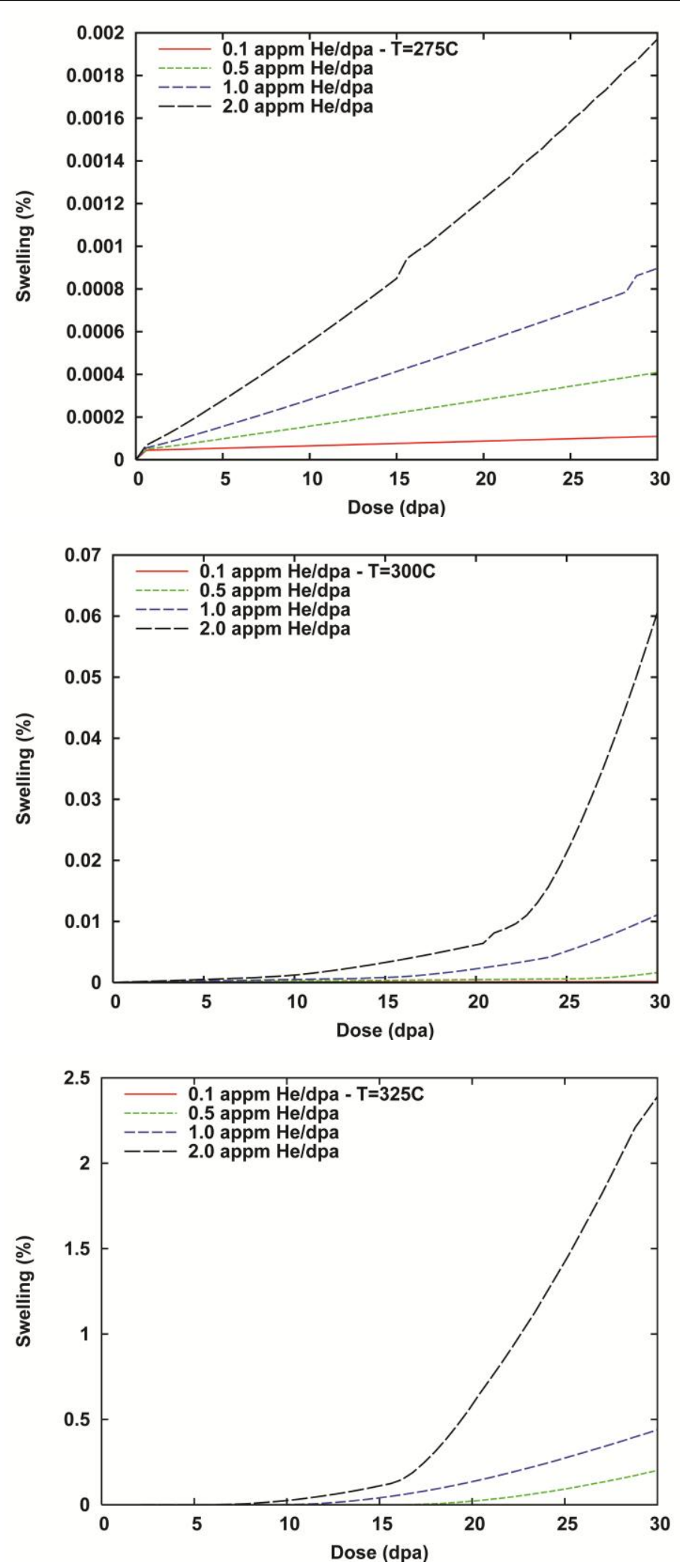

Fig. 8. Fluence dependence of swelling at $\mathrm{T}=275,300$, and $325^{\circ} \mathrm{C}$ for the indicated He/dpa ratios. 
the temperature and dose-rate range of interest to LWR components. However, there are data and calculations that suggest the incubation time may be reduced at lower displacement rates needs to be considered, particularly for the case of BWRs.

The ability to experimentally access the required irradiation conditions is limited because of the very long exposure times. Much of the LWR fleet has already accumulated 20 to 30 effective full power years of exposure and experiments with nearprototypical damage rates can never catch up with the doses expected at end-of-life with life extension. Moreover, the potential for a dose-rate sensitivity suggests that data from accelerated irradiation experiments should be used with caution when predicting the high dose behavior. In this case a well-validated mechanistic microstructural model such that being developed under this project can make a valuable contribution to assessing the swelling to be expected. The model can be used in concert with accelerated irradiations to make the best possible assessment of the irradiation response of the components of interest.

\section{Future Work}

The near-term focus of this modeling work will be focused on list of five required areas for updating the model listed above. In addition, further consultation with other researchers involved in the Light Water Reactor Sustainability Program will be carried out to better determine the expected irradiation exposure conditions for specific BWR and PWR components. This is necessary to establish best estimates for both the displacement rate and irradiation temperature, as well as the primary radiation damage source terms in the model which are dependent on the neutron energy spectrum. An evaluation of the differences between Types 304 and 316 stainless steel will be included to better determine how the compositional differences influence the material parameters used in the models.

A primary modeling objective in the next fiscal year is to incorporate the new nucleation model into the more comprehensive microstructural code. This will greatly improve the cavity evolution component of the larger model and enable the evolving microstructure to provide direct feedback to the nucleation component. This will involve a major restructuring of the code logic and the use of the "dlsode" package [24] used to integrate the many differential equations involved. This effort should require about six months to complete.

A unique aspect of the comprehensive microstructural model is its use of a multicomponent dislocation evolution model while simultaneously simulating cavity evolution. This enables the model to capture the complex mutual interactions that drive radiationinduced microstructural evolution. The second major code update will involve revising the grouping approach used to describe the evolution of the dislocation loop size distribution; the improved grouping scheme discussed above for the bubble size distributions [22] will be adopted to improve point defect conservation in the size distribution. This will also involve changes to code logic and use of dlsode and require about six months of effort.

At the same time, the equations describing the kinetics of point defects and small 
point defect clusters will be revised to enable assessment of both one-dimensional and three-dimensional diffusion as observed in molecular dynamics simulations [20]. This more complex description of point defect and sink interactions may be particularly important at the lower temperatures obtained in LWR components where the sink densities tend to be very high and even modest changes in the point defect kinetics may lead to dramatic changes in the microstructure. This should require about four months of effort.

Finally, it is anticipated that the computational complexity of the updated model may require the use of parallel solver for differential equations. Preliminary discussions have been held with relevant computational scientists and a parallel implementation of the code should be possible by the end of FY14. 


\section{References}

1. C. Cawthorne and E. J. Fulton, "The Nature of Small Defect Clusters," Proc. of Int. Conf., AERE-R5269, AERE Harwell, 1966, pp. 446-454.

2. C. Cawthorne and E. J. Fulton, Nature 216 (1967) 575-576.

3. The body of relevant references is too broad to list here, two helpful recent reviews are: (a) F. A. Garner, "Radiation Damage in Austenitic Steels," Volume 4, pp. 33-95; and (b) P. J. Maziasz and J. T. Busby, "Properties of Austenitic Steels for Nuclear Reactor Applications," Volume 2, pp. 267-283, both in Comprehensive Nuclear Materials, R. J. M.Konings, T. R. Allen, R. E. Stoller, and S. Yamanaka, Eds., Elsevier Ltd., Amsterdam, 2012.

4. R. E. Stoller, "Microstructural Evolution in Fast-Neutron-Irradiated Austenitic Stainless Steels," Ph. D. Thesis, University of California, Santa Barbara, 1987.

5. F. A. Garner, "Void Swelling and Irradiation Creep in Light Water Reactor Environments," in Understanding and Mitigating Ageing in Nuclear Power Plants, Woodhead Publishing Limited, Oxford, 2010, pp. 308-356.

6. R. E. Stoller and G. R. Odette, "A Comparison of the Relative Importance of Helium and Vacancy Accumulation in Void Nucleation," Radiation-Induced Changes in Microstructure, 13th International Symposium, ASTM STP 955, F. A. Garner, N. H. Packan and A. S. Kumar, Editors, American Society of Testing and Materials, Philadelphia, 1987, pp. 358-370.

7. D. M. Stewart, Yu. N. Osetsky, R. E. Stoller, S. I. Golubov, T. Seletskaia, P. J. Kamenski, Phil. Mag. 90 (2010) 935-944.

8. R. E. Stoller and G. R. Odette, J. Nucl. Mater. 131 (1985) 118-125.

9. R. E. Stoller and G. R. Odette, J. Nucl. Mater. 186 (1992) 203-205.

10. R. E. Stoller and L. R. Greenwood, J. Nucl. Mater. 271 \& 272 (1999) 57-62.

11 K. R. Thoms, "Irradiation Research Capabilities at HFIR and ANS," in Proceedings of DOE Workshop on Radiation Effects on Materials in High Radiation Environments, CONF-9008136-1, 1990, see also High Flux Isotope Reactor User Guide, Oak Ridge National Laboratory, May, 2011.

12. L. B. Miller and R. E. Jarka, "Local Modification Of Irradiation Conditions," ANL/EBR035, EBR-II Project Office, Argonne National Laboratory, Idaho Falls, Idaho, 1971.

13. R. E. Stoller, P. J. Maziasz, A. F. Rowcliffe and M. P. Tanaka, J. Nucl. Mater. 155157 (1988) 1328-1334.

14. F. A. Garner, L. R. Greenwood and D. L. Harrod, "Potential High Fluence Response of Pressure Vessel Internals Constructed from Austenitic Stainless Steels', in Sixth Intern. Symp. on Environmental Degradation of Materials in Nuclear Power Systems - Water Reactors, TMS, Warrandale, 1993, pp. 783-790.

15. L. E. Thomas, D. Edwards, K. Asano, S. Ooki and S. Bruemmer, 'Crack-Tip Characteristics in BWR Service Conditions, 10th International Conference on Environmental Degradation of Materials in Nuclear Power Systems - Water Reactors, 2001, issued on CD format, no page numbers. 
16. D. J. Edwards, E. P. Simonen, F. A. Garner, L. R. Greenwood, B. A. Oliver and S. M. Bruemmer, J. Nucl. Mater. 317 (2003) 32-45.

17. D. J. Edwards, F. A. Garner, S. M. Bruemmer, and P. Efsing, J. Nucl. Mater. 384 (2009) 249-255.

18. S. I. Porollo, A. M. Dvoriashin, Yu. V. Konobeev, A. A. Ivanov, S. V. Shulepin and F. A. Garner, J. Nucl. Mater. 359 (2006) 41-49.

19. R. E. Stoller and G. R. Odette, "A Composite Model of Microstructural Evolution in Austenitic Stainless Steel Under Fast Neutron Irradiation," Radiation-Induced Changes in Microstructure, 13th International Symposium, ASTM STP 955, F. A. Garner, N. H. Packan and A. S. Kumar, Editors, American Society of Testing and Materials, Philadelphia, 1987, pp. 371-392.

20. R. E. Stoller, "Primary Radiation Damage Formation," in Comprehensive Nuclear Materials, R. J. M. Konings, T. R. Allen, R. E. Stoller, and S. Yamanaka, Editors, Elsevier Ltd., Amsterdam, 2012, pp. 293-332..

21. S. I. Golubov, A. V. Barashev, and R. E. Stoller, "Radiation Damage Theory," in Comprehensive Nuclear Materials, R. J. M. Konings, T. R. Allen, R. E. Stoller, and S. Yamanaka, Editors, Elsevier Ltd., Amsterdam, 2012, pp. 357-391.

22. S.I. Golubov, A.M. Ovcharenko, A.V. Barashev, B.N. Singh, Phil. Mag. A 81 (2001) 643.

23. S. I. Golubov, R.E. Stoller, S.J. Zinkle, A.M. Ovcharenko, J. Nucl. Mater. 361 (2007) 149-159.

24. A. C. Hindmarsh, SIGNUM, Newsletter of the A.C.M. 15 (1980) 10-11. 